\title{
Comparison of 3T Intracranial Vessel Wall MRI Sequences
}

\author{
(D) A. Lindenholz, (D)A.A. Harteveld, (D).J.M. Zwanenburg, (D).C.W. Siero, and (D). Hendrikse
}

\begin{abstract}
BACKGROUND AND PURPOSE: Intracranial vessel wall MR imaging plays an increasing role in diagnosing intracranial vascular diseases. For a complete assessment, pre- and postcontrast sequences are required, and including other sequences, these result in a long scan duration. Ideally, the scan time of the vessel wall sequence should be reduced. The purpose of this study was to evaluate different intracranial vessel wall sequence variants to reduce scan duration, provided an acceptable image quality can be maintained.
\end{abstract}

MATERIALS AND METHODS: Starting from the vessel wall sequence that we use clinically (6:42 minutes), 6 scan variants were tested (scan duration ranging between 4:39 and 8:24 minutes), creating various trade-offs among spatial resolution, SNR, and contrast-to-noise ratio. In total, 15 subjects were scanned on a 3T MR imaging scanner: In 5 subjects, all 7 variants were performed precontrast-only, and in 10 other subjects, the fastest variant (4:39 minutes) and our clinically used variant (6:42 minutes) were performed pre- and postcontrast.

RESULTS: The fastest variant (4:39 minutes) had higher or comparable SNRs/contrast-to-noise ratios of the intracranial vessel walls compared with the reference sequence (6:42 minutes). Qualitative assessment showed that the contrast-to-noise ratio was most suppressed in the fastest variant of 4:39 minutes and the variant of 6:42 minutes pre- and postcontrast. SNRs/contrast-to-noise ratios of the fastest variant were all, except one, higher compared with the variant of $6: 42$ minutes $(P<.008)$. Furthermore, the fastest variant $(4: 39$ minutes) detected all vessel wall lesions identified on the 6:42-minute variant.

CONCLUSIONS: A 30\% faster vessel wall sequence was developed with high SNRs/contrast-to-noise ratios that resulted in good visibility of the intracranial vessel wall.

ABBREVIATIONS: CNR = contrast-to-noise ratio; DANTE = delay alternating with nutation for tailored excitation; PD = proton density; SENSE = sensitivity encoding; VIRTA = volumetric isotropically reconstructed turbo spin-echo acquisition; VISTA = volumetric isotropic turbo spin-echo acquisition

$\mathrm{H}^{\mathrm{i}}$ igh-resolution intracranial vessel wall MR imaging plays an increasing role in diagnosing intracranial vascular diseases. ${ }^{1,2}$ The main advantage of this imaging technique compared with lumen-based methods such as CT angiography and digital subtraction angiography is the visualization of the vessel wall itself, including the detection of vessel wall lesions that do not necessarily show (or only subtle) luminal narrowing. ${ }^{3-7}$ Intracranial

Received March 7, 2017; accepted after revision February 17, 2018

From the Department of Radiology (A.L., A.A.H., J.J.M.Z., J.C.W.S., J.H.) University Medical Center Utrecht, Utrecht, the Netherlands; and Spinoza Center for Neuroimaging (J.C.W.S.), Amsterdam, the Netherlands.

This work was supported by the European Research Council grant No. 637024 (J.H.).

Please address correspondence to A. Lindenholz, MD, Department of Radiology, University Medical Center Utrecht, Postbox 85500, 3508 GA Utrecht, the Netherlands; e-mail: A.Lindenholz@umcutrecht.nl

- Indicates open access to non-subscribers at www.ajnr.org

$\equiv$ Indicates article with supplemental on-line tables.

$\square$ Indicates article with supplemental on-line photos.

http://dx.doi.org/10.3174/ajnr.A5629 vessel wall imaging can be used for the detection and characterization of plaque burden in intracranial atherosclerotic disease, which is known to be one of the most important contributing factors to ischemic stroke and may be detected at an early stage. ${ }^{8-10}$ It can also be helpful in the differentiation of other vascular diseases, such as vasculitis and reversible vasoconstriction syndrome, with, for instance, visualization of vessel wall enhancement. ${ }^{11}$ Furthermore, intracranial vessel wall imaging may aid in the diagnosis of aneurysm rupture and intracranial dissection, though this is less supported in the literature. ${ }^{12-14}$

Intracranial vessel wall MR imaging requires high spatial resolution to visualize the thin vessel wall and potential accompanying vessel wall lesions. Currently, most 3D-acquired vessel wall sequences use a voxel size between 0.4 and $0.7 \mathrm{~mm}$, though this is larger than the normal diameter of the intracranial vessel walls. ${ }^{2,15}$ In addition, a high signal-to-noise ratio and contrast-to-noiseratio (CNR) are required to delineate the vessel wall from surrounding tissue (ie, blood, CSF, and parenchyma). ${ }^{1,2,16}$ At higher magnetic field strengths, a higher spatial resolution and/or SNR 
Table 1: Scan parameters of the acquired scan variants

\begin{tabular}{|c|c|c|c|c|c|c|c|}
\hline & $\begin{array}{l}\text { TIWI VISTA }{ }^{d} \\
\text { Variant } 1\end{array}$ & $\begin{array}{l}\text { PD }_{w} \text { VISTA } \\
\text { Variant } 2^{\mathrm{a}}\end{array}$ & $\begin{array}{l}\text { TIWI VIRTA } \\
\text { Variant }^{\text {d }}{ }^{\text {b }}\end{array}$ & $\begin{array}{l}\text { TIWI VIRTA } \\
\text { Variant } 4\end{array}$ & $\begin{array}{l}\text { TIWI VISTA } \\
\text { Variant } 5^{c}\end{array}$ & $\begin{array}{l}\text { TIWI VISTA } \\
\text { Variant } 6\end{array}$ & $\begin{array}{l}\text { TIWI VIRTA } \\
\text { Variant } 7\end{array}$ \\
\hline Scan duration (min) & $8: 24$ & $7: 50$ & $6: 42$ & $6: 01$ & $5: 52$ & $5: 49$ & $4: 39$ \\
\hline TR/TE (ms) & $1500 / 38$ & $2000 / 40$ & $1500 / 37$ & $1500 / 40$ & $1500 / 40$ & $1500 / 38$ & $1500 / 40$ \\
\hline $\mathrm{FOV}\left(\mathrm{mm}^{3}\right)$ & $200 \times 166 \times 45$ & $200 \times 166 \times 45$ & $200 \times 166 \times 45$ & $200 \times 166 \times 45$ & $200 \times 166 \times 45$ & $200 \times 166 \times 45$ & $200 \times 166 \times 45$ \\
\hline Acquired voxel $\left(\mathrm{mm}^{3}\right)$ & $0.5 \times 0.5 \times 0.5$ & $0.5 \times 0.5 \times 0.5$ & $0.6 \times 0.6 \times 1.0$ & $0.5 \times 0.5 \times 1.0$ & $0.5 \times 0.5 \times 0.5$ & $0.6 \times 0.6 \times 0.6$ & $0.5 \times 0.5 \times 1.0$ \\
\hline Reconstructed voxel $\left(\mathrm{mm}^{3}\right)$ & $0.5 \times 0.5 \times 0.5$ & $0.5 \times 0.5 \times 0.5$ & $0.5 \times 0.5 \times 0.5$ & $0.5 \times 0.5 \times 0.5$ & $0.5 \times 0.5 \times 0.5$ & $0.6 \times 0.6 \times 0.6$ & $0.5 \times 0.5 \times 0.5$ \\
\hline Oversample factor & 1.8 & 1.2 & 1.8 & 1.8 & 1.2 & 1.8 & 1.8 \\
\hline SENSE reduction factor & 1.5 & 2 & 1.5 & 2 & 2 & 1.5 & 2 \\
\hline Overcontiguous slices & No $(90)$ & No $(90)$ & Yes (90) & Yes (90) & No $(90)$ & No (75) & Yes (90) \\
\hline TSE + startup echoes & $56+3$ & $60+5$ & $56+5$ & $56+5$ & $60+5$ & $56+4$ & $56+5$ \\
\hline Turbo direction & Radial & Radial & Y-axis & Radial & Radial & Radial & Radial \\
\hline Refocusing control $\left(\alpha_{\min } / \alpha_{\max }\right)$ & $30 / 120$ & $50 / 120$ & $25 / 120$ & $50 / 120$ & $50 / 120$ & $30 / 120$ & $50 / 120$ \\
\hline Readout bandwidth $(\mathrm{Hz})$ & 607.6 & 358.6 & 732.1 & 360.1 & 358.6 & 753 & 360.1 \\
\hline Reference tissue $\mathrm{T} 1 / \mathrm{T} 2$ (ms) & $1200 / 80$ & $1200 / 100$ & $1200 / 100$ & $1200 / 100$ & $1200 / 100$ & $1200 / 80$ & $1200 / 100$ \\
\hline Anti-DRIVE & Yes & No & Yes & No & No & Yes & Yes \\
\hline Elliptic $k$-space shutter & Yes & Yes & No & No & Yes & Yes & Yes \\
\hline
\end{tabular}

Note: $-\alpha_{\min } / \alpha_{\max }$ indicates $\alpha$ minimum and maximum; $\mathrm{PD}_{\mathrm{W}}$, proton density-weighted; Anti-DRIVE, anti-driven equilibrium.

${ }^{\text {a }}$ Adjusted from Qiao et al. ${ }^{19}$

${ }^{\mathrm{b}}$ Currently used in our clinic.

${ }^{c}$ Adjusted from Qiao et al ${ }^{19}$ with a shorter TR.

d Philips Healthcare.

can be achieved. Therefore, vessel wall MR imaging is currently performed at 3T and higher field strengths. However, with high spatial resolution, the SNR is still limited and the total scan duration is long. Recently published intracranial vessel wall MR imaging sequences have scan durations ranging from 5.0 to 10.2 minutes. ${ }^{16-22}$ To assess vessel wall lesions, one needs pre- and postcontrast acquisitions to evaluate contrast enhancement of the vessel walls. For a complete examination, other sequences, such as time-of-flight MR angiography, diffusion-weighted imaging, and T2 fluid-attenuated inversion recovery images may also be needed, resulting in a long total scan duration. With longer scan durations, motion artifacts may increase, especially in neurologically impaired patients. Ideally, total scan duration of the preand postcontrast vessel wall sequence should be reduced without sacrificing image quality. In this study, SNR and CNR of 1 earlier reported intracranial vessel wall sequence ${ }^{23,24}$ were compared with 6 variations, which include another previously reported sequence, ${ }^{19}$ with different trade-offs among scan duration, resolution, and contrast. Subsequently, pre- and postcontrast images of the fastest vessel wall variant were compared with the current variant used in our daily clinical practice. ${ }^{24}$

\section{MATERIALS AND METHODS Participants}

Fifteen subjects were included in this study. In 5 subjects ( 4 men; mean age, 37 years; range, 28-54 years), all 7 scan variants were performed (precontrast only). Additionally, precontrast and postcontrast comparisons were performed in 10 other subjects ( 9 men; mean age, 53 years; range, 24-68 years). Approval for this study was obtained from the institutional review board of University Medical Center Utrecht. The 10 volunteers who received contrast agent were part of the Posterior Intracranial Vessel Wall Imaging (PIVI) and Intracranial Vessel Wall Imaging (IVI) studies (NTR5688 and NTR2119, respectively, www.trialregister.nl). All subjects gave written informed consent.

\section{Imaging Protocol}

We used a 3T MR imaging system (Achieva; Philips Healthcare, Best, the Netherlands) with an 8-channel phased array sensitivity encoding (SENSE) head coil. All vessel wall sequences consisted of a $3 \mathrm{D}$ fast turbo spin-echo sequence and NEX of 1 . Starting from our earlier described vessel wall sequence (variant 3 in Table 1), ${ }^{23}$ 6 different variants were created, of which one is previously described (variant 2). ${ }^{19}$ In the scan protocol of the precontrast-only acquisitions, the sequence acquisition order was equal in each subject (consecutively, variants 2, 5, 3, 4, 7, 1, and 6; Table 1). Variant 3 was used as a reference sequence for the 6 different precontrast-only acquisitions. Various trade-offs were created among contrast (TR, antidriven equilibrium), spatial resolution (voxel-size, elliptic $k$-space shutter), SNR (oversample factor in the phase-encoding direction, SENSE reduction factor, readout bandwidth, overcontiguous [overlapping] slices, TSE-train flip angle refocusing control), and scan duration (Table 1). All vessel wall scans were repeated with the radiofrequency and gradient turned off for sampling noise images from the receiver coil elements, including image-reconstruction effects such as elliptic $k$ space shutter and parallel (SENSE) reconstruction sensitivities. The noise images were accelerated by leaving out the delay after each TSE train to the next (turned off) excitation. The total scan session took 65 minutes and included the preparation phase, TOF-MRA (acquired voxel size, $0.4 \times 0.7 \times 1.0 \mathrm{~mm}^{3}$; reconstructed voxel size, $0.36 \times$ $0.36 \times 0.5 \mathrm{~mm}^{3}$; acquisition time, 5:12 minutes), and the 7 vessel wall scan variants, including their corresponding noise images. For the vessel wall sequences, the FOV was planned in a transverse/oblique orientation, which covered all large cerebral arteries of the circle of Willis.

In addition, in 10 other subjects, precontrast and postcontrast images were acquired of our earlier published and clinically used variant 3 , which had good CSF suppression, and the fastest, variant 7 (consecutively, variants 3 and 7 in 8 subjects and consecutively, variants 7 and 3 in 2 subjects). We have chosen to compare the fastest variant, variant 7 , with our clinically used variant for clinical and practical purposes. Before acquisition of the postcontrast sequences, a gadolinium-containing contrast agent (gadobutrol, Gadovist, $1.0 \mathrm{mmol} / \mathrm{mL}$; Bayer Schering Pharma, Berlin, Germany) was administered intravenously. The total scan session took 45 minutes and included, consecutively, the preparation 

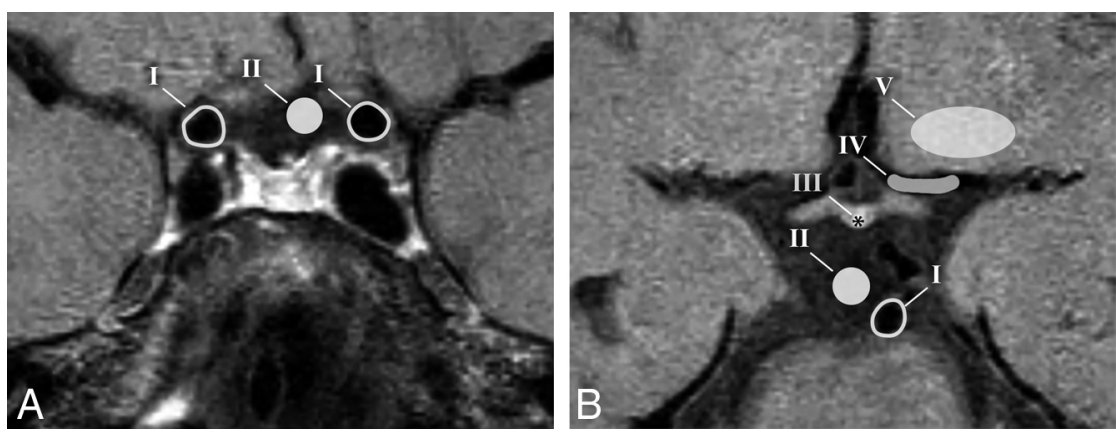

location and configuration (eccentric or concentric) and contrast enhancement, using methods previously described. ${ }^{24,26}$ All scans were blinded and randomly ordered before assessment. When a lesion was found in only one of the variants, the location was re-examined in the other variant to check whether the lesion could be identified retrospectively. Furthermore, the vessel wall images were cross-correlated with the TOF-MRA images for a correct interpretation of the specific arteries, the potential vessel wall lesions, and the lumen of the artery. Included vessels were the anterior cerebral arteries (A1 and A2 segments), distal intracranial internal carotid arteries (clinoid or C5 and cavernous or C4 segments), middle cerebral arteries cumferential of both carotid arteries (I) was used as a marker for the carotid vessel wall that runs through the suprasellar cistern, which was used as marker for CSF (II). B, The circumferential of the basilar artery (I) was used as marker for the basilar vessel wall that runs through the pontine cistern, which was used as second marker for CSF (II). For the pituitary stalk, a homogeneous hyperintense part of the center was used (III). In the lumen of the middle cerebral artery, an ROI was drawn as a marker for blood (IV), and in the left orbital gyrus, an ROI was drawn as marker for brain tissue (V). The ROIs are drawn for illustrative purposes, and the exact contours may differ in the real measurements, depending on patients' specific anatomies.

phase with the survey scans; TOF-MRA precontrast vessel wall sequences; postcontrast DWI; postcontrast vessel wall sequences (acquired $\sim 20$ minutes after the precontrast vessel wall sequence), including noise images; and finally, a postcontrast T2 FLAIR sequence.

\section{Image Analysis}

The mean signal and $\mathrm{SD}$ of the noise $\left(\mathrm{SD}_{\text {Noise }}\right)$ were obtained from the axial plane of the vessel wall images and noise images (magnitude), respectively. ROIs for the mean signal $\left(\right.$ mean $\left._{\mathrm{ROI}}\right)$ were manually drawn as follows: CSF was marked in the suprasellar and pontine cisterns for the intracranial internal carotid arteries and basilar artery, respectively; blood was marked in the lumen of the middle cerebral artery; brain tissue was marked in the left orbital gyrus; and the vessel wall was represented by the circumferential of the intracranial internal carotid arteries (left and right) and basilar artery (within the same slice number as the ROIs for CSF, Fig 1). In the 10 subjects who received contrast agent injection, a homogeneous hyperintense part of the center of the pituitary stalk was used as the ROI to compute the mean as a consistent marker for contrast enhancement (Fig 1). To compute the SD of the noise images, a larger circular ROI was drawn on the noise images, encompassing the ROIs used to compute the mean. The $\mathrm{SNR}$ was calculated as $\mathrm{SNR}=\mathrm{Mean}_{\mathrm{ROI}} / \mathrm{SD}_{\text {Noise }}$ for each subject and subsequently averaged for all subjects included in the comparison. The CNRs were calculated as $\mathrm{CNR}_{\mathrm{x}-\mathrm{y}}=\mathrm{SNR}_{\mathrm{x}}-$ $\mathrm{SNR}_{\mathrm{y}}$. As a measure of motion, vessel wall sequences were coregistered to the prior acquired sequence to calculate the registration parameters ( $\Delta$ Rotation and $\Delta$ Translation), using the Elastix toolbox in MeVisLab (Version 2.7; MeVis Medical Solutions, Bremen, Germany). ${ }^{25}$

An expert neuroradiologist (J.H., with $>15$ years of experience), specialized in intracranial vessel wall imaging, assessed both pre- and postcontrast vessel wall images in multiple planes for image quality, which includes the visibility of the arterial vessel wall, the suppression of blood and CSF, and the existence of artifacts (slow-flow, motion, and free induction decay artifacts). The images of the 10 subjects who received contrast agent injection were also assessed for the presence of vessel wall lesions, including
(M1, M2, and M3 segments), posterior cerebral arteries (P1 and P2 segments), the distal intracranial basilar artery, and distal intracranial vertebral arteries.

\section{Statistical Analysis}

All statistical comparisons were conducted using SPSS statistics (Version 21; IBM, Armonk, New York). Pairwise comparisons were performed between the clinically relevant SNRs and CNRs of the reference variant and the 6 other variants using generalized estimating equations to account for repeated measures on the same subjects. A Friedman test was performed for differences in SNR and CNR between both precontrast and postcontrast images of variants 3 and 7. A post hoc analysis with the Wilcoxon signed rank test was conducted with a Bonferroni correction applied to correct for multiple comparisons. A $P$ value $<.008$ was considered significant.

\section{RESULTS}

All 15 subjects underwent the MR imaging successfully. The quality of all images was sufficient to be used for analysis. Image assessment was not hampered by motion artifacts in any of the scanned subjects.

\section{Comparison of all 7 Scan Variants}

Typical images of the 7 vessel wall scan variants are shown in Fig 2 (axial plane), Fig 3 (sagittal plane), and On-line Fig 1 (coronal plane). The images show clear differences among the variants in contrast and visibility of the vessel wall. Qualitative assessment of the images in the axial plane shows poor visibility of the vessel wall in variants 1 (8:24 minutes) and 6 (5:49 minutes), acquired with an isotropic voxel size. Variants 4 (6:01 minutes) and 7 (4:39 minutes), which were acquired with an anisotropic voxel size, had higher qualitative assessment scores compared with the other variants. Qualitative assessment of the axial, sagittal, and coronal images showed that CSF was most suppressed in variants 3 (6:42 minutes) and 7 (4:39 minutes). These 2 variants are compared before and after contrast administration in the next paragraph (Table 2). Variant 1 (8:24 minutes) also had an adequate CSF 

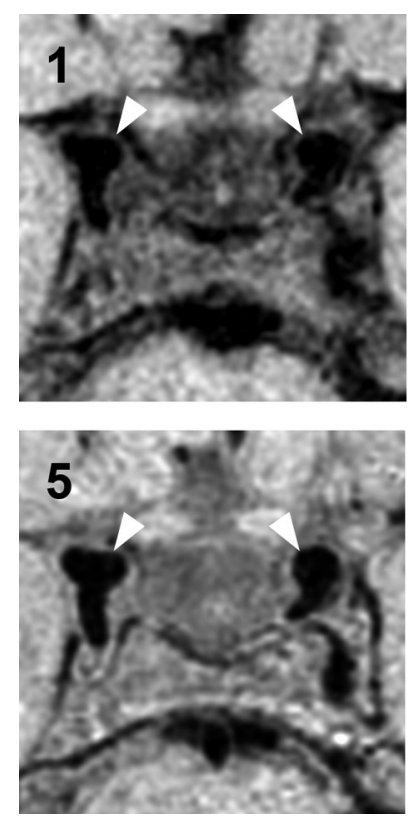
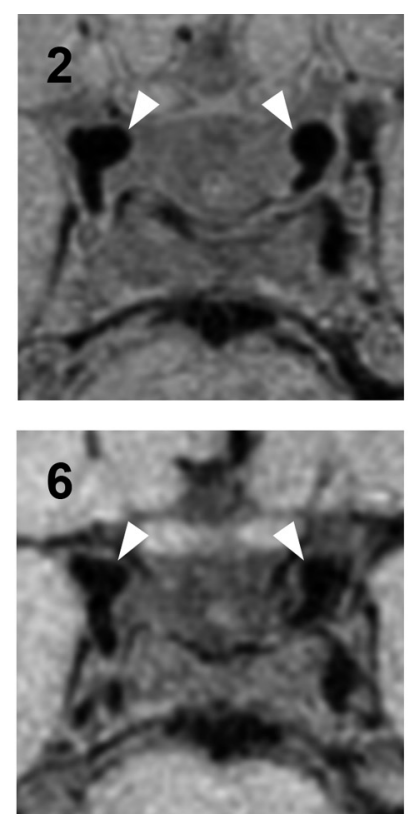
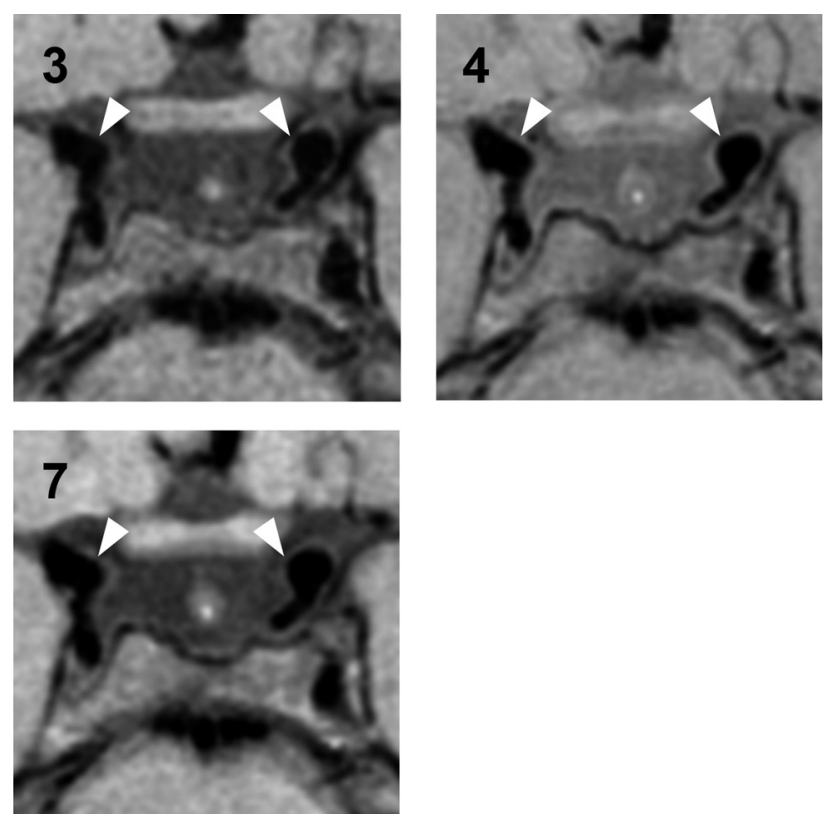

FIG 2. Sample images in the axial plane of the 7 different scan variants performed at $3 T$ (precontrast, in order of decreasing scan duration). Both distal intracranial internal carotid arteries (white arrowheads) with the bifurcation of the posterior communicating artery are depicted, surrounded by CSF. 1, TIWI VISTA variant 1 (8:24 minutes). 2, Proton density-weighted VISTA variant 2 (7:50 minutes), adjusted from Qiao et al. ${ }^{19}$ 3, TIWI VIRTA variant 3 (6:42 minutes). 4, TTWI VIRTA variant 4 (6:01 minutes). 5, TIWI VISTA variant 5 (5:52 minutes), adjusted from Qiao et al ${ }^{19}$ with a shorter TR. 6, TIWI VISTA variant 6 (5:49 minutes). 7, TIWI VIRTA variant 7 (4:39 minutes).
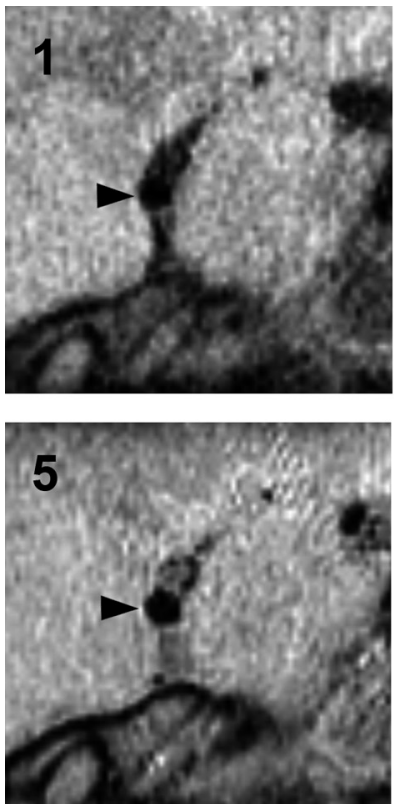
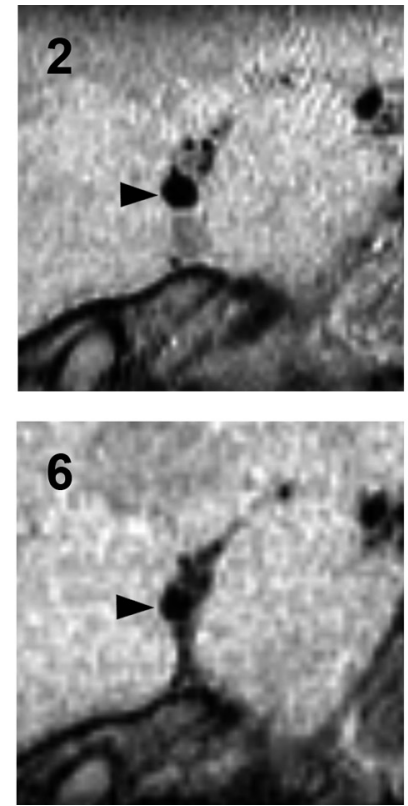
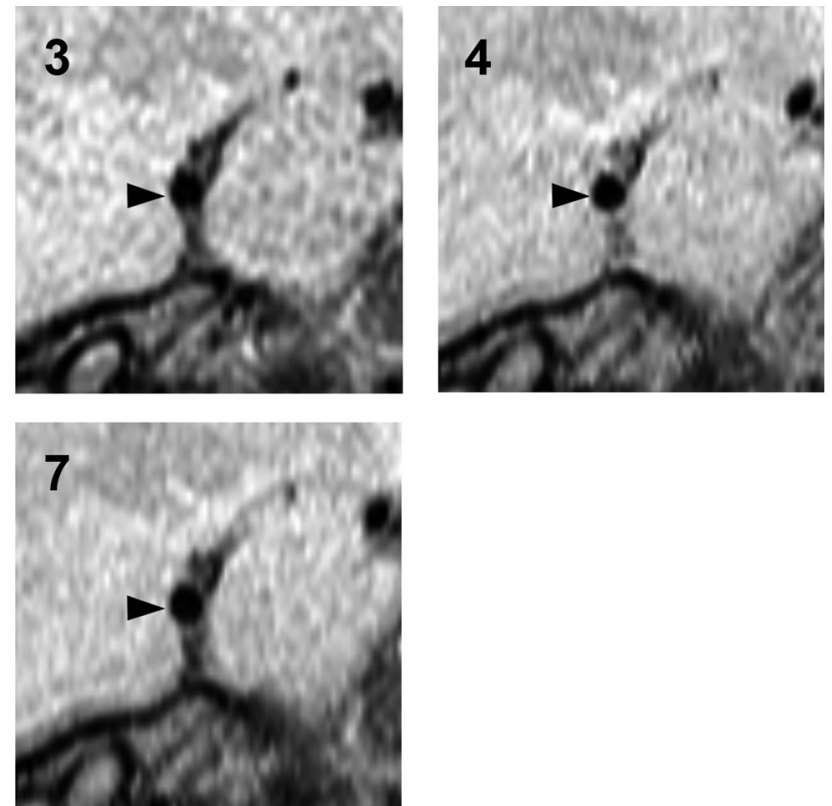

FIG 3. Sample images in the sagittal plane of the 7 different scan variants performed at $3 T$ (precontrast, in the order of decreasing scan duration). The right MCA (black arrowheads) is depicted in all images, surrounded by CSF and brain parenchyma. 1, TIWI VISTA variant 1 (8:24 minutes). 2, Proton density-weighted VISTA variant 2 (7:50 minutes), adjusted from Qiao et al. ${ }^{19} 3$, TTWI VIRTA variant 3 (6:42 minutes). 4, TIWI VIRTA variant 4 (6:01 minutes). 5, TIWI VISTA variant 5 (5:52 minutes), adjusted from Qiao et al ${ }^{19}$ with a shorter TR. 6, TIWI VISTA variant 6 (5:49 minutes). 7, TIWI VIRTA variant 7 (4:39 minutes).

suppression, but the axial and sagittal images had an overall granular appearance, which made assessment and delineation of the vessel wall with the surrounding CSF challenging. This granular appearance was also seen to a lesser extent in variants 5 (5:52 minutes) and 6 (5:49 minutes), but with worse CSF suppression. In the axial plane, the vessel wall was best seen in variants 2 (7:50 minutes), 3 (6:42 minutes), 4 (6:01 minutes), and 7 (4:39 min- utes). In both the sagittal and coronal planes, the vessel wall is of the highest quality in the isotropic variant 2 (7:50 minutes), though the contrast with the surrounding CSF is less, which makes delineation of the outer margins of the vessel wall more difficult. Due to the granular appearance, the perpendicular visibility (sagittal plane) of the middle cerebral artery was poor in variants 1 (8:24 minutes) and 6 (5:49 minutes). In the aniso- 
Table 2: SNRs and CNRs of the precontrast and postcontrast scan variants 3 and $7^{\mathrm{a}}$

\begin{tabular}{|c|c|c|c|c|c|c|}
\hline & \multicolumn{2}{|c|}{ Precontrast } & \multirow[b]{2}{*}{$P$ Value } & \multicolumn{2}{|c|}{ Postcontrast } & \multirow[b]{2}{*}{$P$ Value } \\
\hline & $\begin{array}{l}\text { TIWI VIRTA } \\
\text { (Variant 3) }^{f}\end{array}$ & $\begin{array}{l}\text { TIWI VIRTA } \\
\text { (Variant 7) }\end{array}$ & & $\begin{array}{l}\text { TIWI VIRTA } \\
\text { (Variant 3) }^{\text {f }}\end{array}$ & $\begin{array}{l}\text { TIWI VIRTA } \\
\text { (Variant 7) }\end{array}$ & \\
\hline $\mathrm{SNR}_{\text {tissue }}{ }^{\mathrm{b}}$ & $28.0 \pm 1.8$ & $40.5 \pm 3.4$ & $.005^{g}$ & $27.9 \pm 1.3$ & $42.4 \pm 2.8$ & $.005^{g}$ \\
\hline $\mathrm{SNR}_{\text {carotid vessel wall }}{ }^{\mathrm{C}}$ & $15.3 \pm 1.4$ & $20.8 \pm 2.7$ & $.005^{8}$ & $17.2 \pm 0.9$ & $26.4 \pm 4.0$ & $.005^{g}$ \\
\hline$S N R_{\text {basilar vessel wall }}$ & $13.0 \pm 1.4$ & $15.9 \pm 1.7$ & $.005^{g}$ & $13.4 \pm 2.4$ & $19.2 \pm 1.8$ & $.005^{g}$ \\
\hline $\mathrm{SNR}_{\text {suprasellar CSF }}$ & $9.1 \pm 2.0$ & $11.9 \pm 2.2$ & $.007^{\mathrm{g}}$ & $11.3 \pm 0.7$ & $14.1 \pm 2.4$ & .013 \\
\hline$S_{N R}$ pontine CSF & $5.9 \pm 2.1$ & $8.0 \pm 2.0$ & $.007^{g}$ & $6.5 \pm 1.8$ & $11.2 \pm 2.6$ & $.007^{g}$ \\
\hline $\mathrm{SNR}_{\text {blood }}$ & $3.6 \pm 0.6$ & $5.4 \pm 1.2$ & $.005^{\mathrm{g}}$ & $3.8 \pm 0.5$ & $6.3 \pm 1.4$ & $.007^{g}$ \\
\hline$S N R_{\text {pituitary gland }}$ & $23.0 \pm 3.4$ & $33.4 \pm 4.7$ & $.005^{g}$ & $33.3 \pm 3.5$ & $49.5 \pm 6.7$ & $.005^{g}$ \\
\hline $\mathrm{CNR}_{\text {carotid vessel wall-CSF }}{ }^{\mathrm{d}}$ & $6.1 \pm 1.6$ & $9.0 \pm 1.5$ & $.005^{g}$ & $6.0 \pm 0.7$ & $12.3 \pm 3.6$ & $.007^{\mathrm{g}}$ \\
\hline $\mathrm{CNR}_{\text {basilar vessel wall-CSF }}$ & $7.2 \pm 1.4$ & $7.9 \pm 1.4$ & .059 & $7.0 \pm 2.0$ & $8.0 \pm 1.9$ & .285 \\
\hline $\mathrm{CNR}_{\text {carotid vessel wall-blood }}{ }^{\mathrm{c}}$ & $11.6 \pm 1.3$ & $15.5 \pm 2.9$ & .013 & $13.5 \pm 1.0$ & $20.1 \pm 3.8$ & $.007^{g}$ \\
\hline
\end{tabular}

${ }^{a}$ Data are mean and SD calculated in 10 subjects. A Wilcoxon signed rank test was used to compare the differences between precontrast variants 3 and 7 and postcontrast variants 3 and 7 (additional statistical comparisons are shown in On-line Table 2).

${ }^{\mathrm{b}}$ Tissue ROI is located at the left orbital gyri.

${ }^{\mathrm{C}}$ The mean of the left and right distal intracranial internal carotid arteries.

d Suprasellar CSF is used as reference.

e Pontine CSF is used as reference.

${ }^{f}$ Currently used in our clinic.

${ }^{8}$ Statistically significant $(P<.008)$
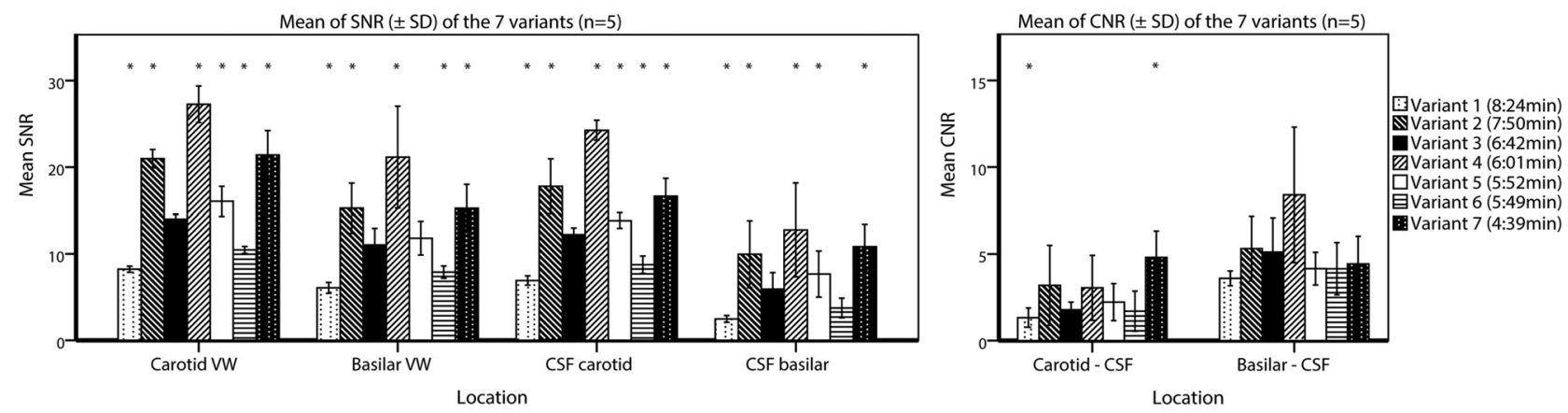

FIG 4. Barplots showing the mean and SD of the clinically relevant SNRs and CNRs of all 7 vessel wall imaging variants. The means and SDs are calculated for 5 subjects The mean of the left and right intracranial internal carotid arteries was used for the carotid vessel wall. The asterisks indicate a significant difference compared with reference variant 3 (corrected $P$ value for multiple comparisons, $P<.008$ ) using generalized estimating equations to account for repeated measures on the same subjects. VW indicates vessel wall.

tropic variants 3 (6:42 minutes), 4 (6:01 minutes), and 7 (4.39 minutes), the vessel wall could be delineated in the sagittal plane, but the overall appearance was blurrier (Fig 3). All variants had sufficient blood suppression without flow artifacts, which could have limited assessment in the large intracranial arteries.

The mean SNRs and CNRs of the clinically most relevant regions (ie, the basilar and carotid vessel walls and the surrounding CSF) are shown in Fig 4, including the statistical comparisons with reference variant 3 . The SNR and, more important, the calculated CNR results for all variants are shown in On-line Table 1. Relatively high SNRs of the vessel wall were measured for variants 2 (7:50 minutes), 4 (6:01 minutes), and 7 (4:39 minutes). In these 3 variants, the SNR of the intracranial internal carotid vessel wall was $>21$, and the SNR of the basilar vessel wall, $>15$. All 7 variants showed good blood signal suppression, which resulted in most variants having a relatively high CNR between blood and the intracranial internal carotid artery vessel wall (CNRs ranging from 5.7 to 23 , On-line Table 1). All except 1 variant performed borderline in the CNRs of the carotid vessel wall and the surrounding CSF (ranging from 1.3 to 3.2). Variant 7 had the highest CNR between the carotid vessel wall and the surrounding CSF (4.8). All variants performed better in the CNRs between the basilar artery vessel wall and CSF, which were comparable in 6 of 7 variants (ranging from 3.6 to 5.3); only variant 4 (6:01 minutes) had a higher CNR (8.4) but also a large SD (3.9, Fig 4).

\section{Comparison of Variant 3 (6:42 minutes) versus 7 (4:39 minutes)}

The average SNRs and CNRs of both precontrast and postcontrast images of variants 3 and 7 are shown in Table 2. Comparing SNRs and CNRs of the precontrast and postcontrast scans (Table 2) for variants 3 and 7 showed that tissue, the basilar vessel wall, suprasellar CSF of only variant 7, pontine CSF, and blood SNR did not differ significantly (all $P$ values $>.008$, On-line Table 2 ), while the SNR of the carotid vessel wall of variant $7(P=.005)$ and the CNRs between the carotid vessel wall and CSF $(P=.005$ and .007 for variants 3 and 7, respectively) tended to be higher for the postcontrast images. Significant differences for the $\mathrm{SNR}_{\text {pituitary gland }}$ were expected because of a strong enhancing pituitary gland on postcontrast images. For both precontrast and postcontrast vessel wall images, the SNRs and CNRs of variant 7 (4:39 minutes) were higher than those of variant 3 (6:42 minutes). These differences were all significant $(P<.008)$, except for the 

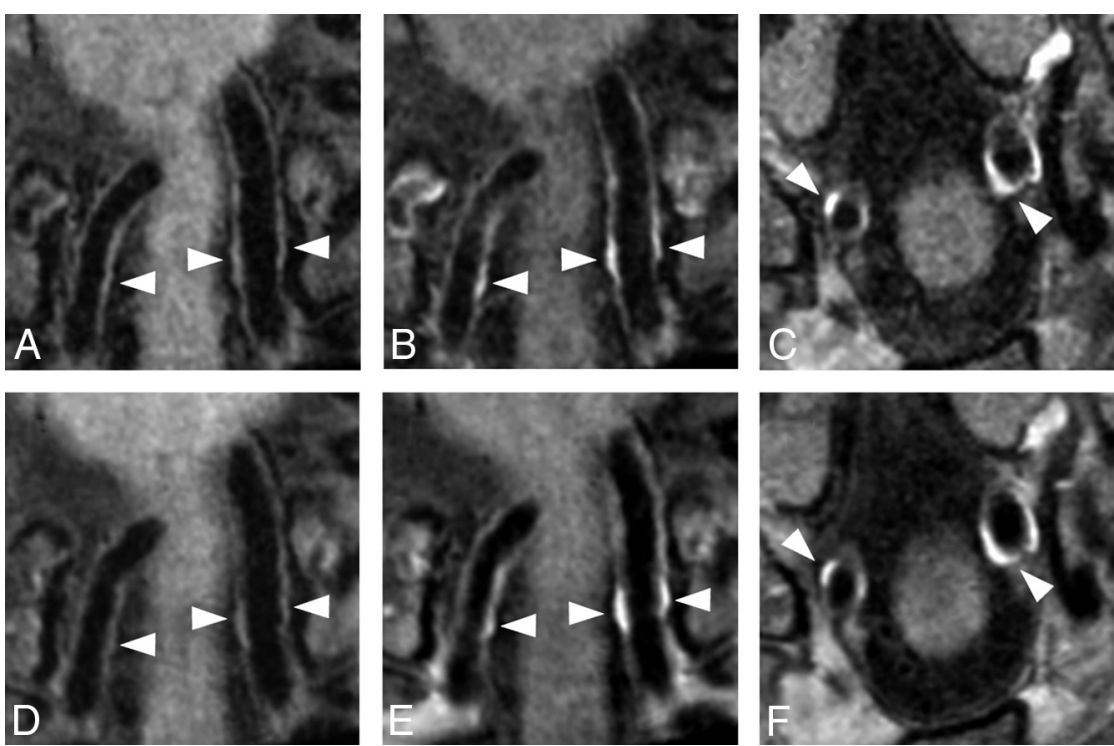

FIG 5. Matching vessel wall lesions of the distal vertebral arteries (white arrowheads) seen before and after contrast with variant 3 (6:42 minutes; $A-C)$ and variant 7 (4:39 minutes; $D-F)$ in a 59-year-old man with multiple cardiovascular risk factors. Subtle (concentric) wall thickening is seen in both the left and right vertebral arteries before contrast $(A$ and $D)$, with clear contrast enhancement on the postcontrast image ( $B$ and $E$ ). Variant 3 (6:42 minutes, $B$ and $C$ ) was acquired approximately 2 minutes after contrast injection, and variant 7 ( $4: 39$ minutes, $E$ and $F$ ), approximately 9 minutes after contrast injection. Some patient motion was seen in the postcontrast series of variant 7. Postcontrast transverse images of variants $3(C)$ and $7(F)$ show clear vessel wall enhancement in both vertebral arteries (white arrowheads).

$\mathrm{SNR}_{\text {suprasellar CSF }}$ of variant 7, the $\mathrm{CNR}_{\text {basilar wall-CSF }}$ of both variants $(P=.059$ and $P=.285)$, and the $\mathrm{CNR}_{\text {carotid vessel wall-blood }}$ of variant $3(P=.013)$ (Table 2$)$.

Qualitative assessment of both precontrast and postcontrast vessel wall images in multiple planes showed that in all subjects, the vessel wall could be delineated for both sequences (Fig 5). Overall, variant 7 (4:39 minutes) was slightly more affected by free induction decay artifacts in the outer regions of the FOV and slow-flow artifacts, but this finding did not affect the assessment of the large intracranial arteries. In 1 subject, variant 7 (4:39 minutes) showed small motion artifacts, but they did not have an influence on the assessment. Sixteen vessel wall lesions were identified in variant 3 (6:42 minutes), and 19 , in variant 7 (4:39 minutes). In 4 of 10 subjects, no vessel wall lesions were detected. Vessel wall lesions (on the basis of the 19 detected vessel wall lesions) were found in the carotid arteries $(n=3)$, middle cerebral arteries $(n=2)$, vertebral arteries $(n=10)$, basilar artery $(n=3)$, and posterior cerebral artery $(n=1)$, of which 12 were enhancing (a full description of all lesions is found in On-line Table 3). All 16 lesions identified in vessel wall variant 3 (6:42 minutes) matched vessel wall lesions identified in variant 7 (4:39 minutes) (Fig 5). One vessel wall lesion was reported as eccentric in variant 3 and concentric in variant 7 , and 1 lesion, as enhancing in variant 3 and nonenhancing in variant 7 . Three lesions were missed with vessel wall variant 3: one lesion located at the bifurcation of the basilar artery $\mathrm{P} 1$, one at the left internal carotid artery, and 1 at the left vertebral artery.

\section{DISCUSSION}

The purpose of this study was to evaluate different variants of MR imaging sequences for intracranial vessel wall imaging to reduce scan duration, provided that an acceptable image quality could be maintained. In the precontrast comparison, the fastest one, variant 7 (4:39 minutes), had a significantly higher CNR between the vessel wall and blood, compared with variant 3, while it was about $30 \%$ faster. Also, the CNR between the vessel wall and CSF was better for variant 7 compared with variant 3 . The favorable SNRs and CNRs of variant 7 also remained in the second comparison in which both precontrast and postcontrast images were acquired and were higher compared with our clinically used variant 3 (Table 2). Especially in the elderly population, the superior contrast between the vessel wall and CSF can be beneficial. In the aging brain, tissue atrophy increases and consequently the intracranial vessels are more richly surrounded by CSF.

The quality of an MR image is a balance of resolution, SNR/CNR, and scan duration. A gain in 1 aspect results in a sacrifice of another. The ideal balance is difficult to determine and depends on the specific application and the clinical question to be answered. Several pulse sequence optimizations have been developed to address these technical challenges. ${ }^{17-19,21,26}$ For high CNR of the vessel wall, both blood and CSF need to be simultaneously suppressed. ${ }^{1} 3 \mathrm{D}$ variable flip angle refocusing pulse sequences are used because of their high intrinsic black-blood properties and the ability to generate multiplanar reformatted images, but they have less effect on the slow-flowing CSF. ${ }^{17,19-22,27}$ For better CSF suppression, proton densityweighted imaging with radial ordering modulation of the $k$-space (short TE), ${ }^{19}$ anti-driven equilibrium, ${ }^{18,23,28}$ or prepulses such as delay alternating with nutation for tailored excitation (DANTE) $)^{17,21,29}$ and inversion recovery ${ }^{30}$ have been proposed.

In 4 of 7 variants, the anti-driven equilibrium technique was used. This technique drives the magnetization back to the negative $\mathrm{M}_{\mathrm{z}}$-axis by further radiofrequency excitation and results in more adequate CSF suppression. ${ }^{18,24,27}$ A second possibility to improve image contrast between the vessel wall and CSF is the use of a DANTE prepulse. ${ }^{17,21,29}$ When one applies DANTE pulse trains, the longitudinal magnetization of static tissue largely remains but flowing spins are suppressed due to a spoiling effect. ${ }^{29}$ This suppression is less for slow-flowing fluid, especially below 0.1 $\mathrm{cm} / \mathrm{s}$; therefore, DANTE will be less useful for CSF suppression around the distal cerebral arteries. The DANTE prepulse also improves the suppression of blood, notably for slow and turbulent flow. Slow flow is a well-known pitfall in the assessment of vessel wall imaging. ${ }^{2}$ In the current study, the postcontrast vessel wall showed a higher SNR/CNR compared with the precontrast vessel wall, which may suggest slow-flow. Another explanation that may have led to higher SNRs of the postcontrast vessel walls is contrast 
absorption into the vessel wall itself. The flow artifacts seen in variant 7 may be a potential problem, and a DANTE prepulse might be a viable addition for more effective blood suppression. A third option to improve the contrast between the vessel wall and surrounding tissue is using inversion recovery. ${ }^{30}$ This technique is mainly applied in 2D acquisitions; however, reports have also been published with $3 \mathrm{D}$ acquisitions on a $7 \mathrm{~T} \mathrm{MR}$ imaging system. ${ }^{31}$

Generally, a lower minimum refocusing angle results in better flow suppression but also in a lower SNR. Because of the higher minimum refocusing angles in the TSE train of vessel wall variants $2,4,5$, and 7 , they may yield more SNRs but probably at the cost of blurring due to a worse point spread function. ${ }^{16,32}$ This may also partly explain the "smoother" appearance in variants 4 and 7 (Fig 2). However, these sequences do show the vessel wall clearly, and in our opinion, radiologists often prefer blurring compared with images with a very narrow point spread function because this results in more noisy images, which are more difficult to assess, even though the true resolution is higher.

A disadvantage of variants 3, 4, and 7 might be the anisotropic resolution in the slice direction, which makes it less beneficial for multiplanar assessment (Figs 2 and 3). ${ }^{1,19}$ In specific clinical situations, multiplanar reconstructions may be required, for example, when assessing lesions in the middle cerebral artery. However, in daily clinical practice, image interpretation can regularly be performed in the axial or oblique plane alone, and in these situations, a nearly isotropic voxel size may be adequate for interpretation of vessel wall images. ${ }^{23,24,33}$ In addition, the scan variants with an anisotropic voxel size have a larger overall voxel size, which results in a higher SNR per voxel, but they also can offer a shorter effective scan time. On the other hand, using an anisotropic voxel size potentially increases partial volume effects compared with the isotropic-derived variants. ${ }^{34}$ Consequently, vessel wall thickness might be overestimated or small lesions might be missed. To reduce the scan duration further, we applied an elliptic $k$-space shutter in 5 variants. Theoretically, this might also reduce the image quality because in enabling the elliptic $k$-space shutter, the outer part of the $k$-space is cut off, leading to a reduction in the effective resolution. However, in practice, the effect may be limited, particularly because the longer scan durations needed for a full acquisition make the images more prone to motion artifacts. Another way to reduce scan duration is the use of $2 \mathrm{D}$ methods as an alternative to $3 \mathrm{D}$ imaging methods. These $2 \mathrm{D}$ methods have anisotropic voxels because the slice thickness is normally much larger than the in-plane voxel size. When these slices are planned perpendicular to the vessels, this is not a problem. However, these $2 \mathrm{D}$ methods have the drawback of small coverage and the need to be focused on a limited vessel wall region. The optimal ROI may be difficult to determine beforehand. ${ }^{26,35}$

Besides several advanced pulse sequence optimization techniques, better hardware may also improve image quality. In the current study, a relatively low number of receiver channels ( 8 channels) was used. Currently, up to 32 channels are commonly used to investigate vessel wall sequences. ${ }^{16,20,27}$ Also, when one uses a higher number of channels in the receiver coil, more possibilities such as parallel imaging might be available to reduce the scan duration without severely compromising image quality.
Moreover, parallel imaging, like SENSE, also performs better at higher field strengths. ${ }^{36}$ Another novel acceleration technique to reduce the scan duration even further may be compressed sensing. ${ }^{37}$ This technique has already shown promising results for extracranial carotid vessel wall imaging. ${ }^{38}$

This study has some limitations. First, the true quality of an MR image is the result of a multidimensional trade-off among numerous parameters. Many of the parameters are also interrelated and may have a combined effect on resolution, SNR/CNR, or scan duration, and it is difficult to compare these parameters one-to-one for true image quality. Furthermore, in the comparison of all 7 variants, the same order of sequences was used, which may have led to more motion artifacts in the later scheduled sequences. However, the extent of movement, measured in $\Delta$ Rotation and $\Delta$ Translation between subsequent sequences, did not increase during the acquisition time (On-line Fig 2). Also, in the second comparison (variant 3 versus 7), contrast enhancement may have been stronger due to a longer absorption time in the latest scheduled variant (in our study mostly variant 7). However, statistical comparison (variants 3 and 7 versus variants 7 and 3) did not show any significant differences (data not shown). This study lacked a systematic comparison among the different parameters to investigate the effect on image quality. Vessel wall variants were created to reduce scan duration with the focus on the development of a clinically usable sequence that can be incorporated into existing scan protocols.

The second limitation is the manually drawn ROIs for measuring the SNR. Although we tried to be consistent in the location, it was not always possible to use the exact same slice number for all variants for the SNR measurement of the vessel wall and CSF, mainly due to intrasubject differences such as patient movement and intersubject differences in planning and anatomy. Furthermore, due to flow effects of CSF in the basal cisterns, a better delineation can be made of the proximal intracranial arteries compared with the more distal intracranial arteries, where the CSF flow is lower. In general, the SNR of the vessel wall may be underestimated because of partial volume effects and difficulties of manually drawing an ROI around the circumferential of the vessel wall. This effect is even more obvious in the thinner basilar artery vessel wall, where the measured SNRs are consistently lower compared with the intracranial internal carotid artery vessel wall with surrounding tissue/CSF. Also, the CSF was often more suppressed in the pontine cistern, most likely due to a faster CSF flow, which resulted in still comparable or even higher CNRs of the basilar artery and CSF compared with the intracranial internal carotid arteries and CSF (Table 2 and On-line Table 1). This partly subjective determination of the circumferential of the vessel walls and CSF is subject to measurement errors. However, the same approach was used in all 7 variants; therefore, it is unlikely that it influenced the relative differences in performance of the compared variants.

Third, no histology or in vivo reference standard for vessel wall imaging of the vessel wall lesions that were identified in this study was available. Therefore, no validation against the ground truth could be performed. Earlier reports already described relatively high numbers of vessel wall lesions or atherosclerotic plaques in asymptomatic subjects. ${ }^{28,39,40}$ In the current study, mostly 
healthy volunteers were used for assessment of the intracranial vessel wall. Larger patient groups, including symptomatic patients, are needed for a more thorough assessment of vessel wall lesion visibility and contrast enhancement and, subsequently, the true utility of the faster vessel wall variants in clinical practice.

\section{CONCLUSIONS}

In this study, a considerably faster clinically feasible vessel wall sequence (4:39-minute scan duration) with high SNRs and CNRs was developed, which resulted in a good visibility of the intracranial vessel wall in the axial plane. Qualitative assessment showed promising results in overall image quality and detecting vessel wall lesions. The faster scan duration allows pre- and postcontrast acquisition of vessel wall images with sufficient remaining scan duration for other imaging sequences in patient studies.

\section{ACKNOWLEDGMENTS}

The authors acknowledge Dr Hugo J. Kuijf from the Image Sciences Institute, University Medical Center Utrecht, for his technical support in coregistering the vessel wall sequences using MeVisLab and Chris van Kesteren from the Radiology Department of the University Medical Center Utrecht, for his support in figure layout.

Disclosures: Arjen Lindenholz-RELATED: Grant: European Research Council, Comments: grant No. 637024, ${ }^{*}$ Anita A. Harteveld—RELATED: Grant: European Research Council under the European Union Horizon 2020 Programme (H2020)/European Research Council grant agreement No. 637024.* Jaco J.M. Zwanenburg— UNRELATED: Grants/Grants Pending: European Research Council starting grant, Comments: funding from the European Research Council under the European Union Seventh Framework Programme (FP7/2007-2013)/European Research Council grant agreement No. 337333. Title of the grant: "Towards Understanding Cerebral Small Vessel Disease: Innovative, MRI-Based, Functional Markers to Discover the Terra Incognita between Large Vessels and Macroscopic Brain Lesions."* *Money paid to the institution.

\section{REFERENCES}

1. Dieleman N, van der Kolk AG, Zwanenburg JJ, et al. Imaging intracranial vessel wall pathology with magnetic resonance imaging: current prospects and future directions. Circulation 2014;130:192201 CrossRef Medline

2. Mandell DM, Mossa-Basha M, Qiao Y, et al. Intracranial vessel wall MRI: principles and expert consensus recommendations of the American Society of Neuroradiology. AJNR Am J Neuroradiol 2017; 38:218-29 CrossRef Medline

3. Qiao Y, Anwar Z, Intrapiromkul J, et al. Patterns and implications of intracranial arterial remodeling in stroke patients. Stroke 2016;47: 434-40 CrossRef Medline

4. Zou XD, Chung YC, Zhang L, et al. Middle cerebral artery atherosclerotic plaques in recent small subcortical infarction: a three-dimensional high-resolution MR study. Biomed Res Int 2015;2015: 540217 CrossRef Medline

5. Yoon Y, Lee DH, Kang DW, et al. Single subcortical infarction and atherosclerotic plaques in the middle cerebral artery: high-resolution magnetic resonance imaging findings. Stroke 2013;44:2462-67 CrossRef Medline

6. Alexander MD, Yuan C, Rutman A, et al. High-resolution intracranial vessel wall imaging: imaging beyond the lumen. J Neurol Neurosurg Psychiatry 2016;87:589-97 CrossRef Medline

7. Bodle JD, Feldmann E, Swartz RH, et al. High-resolution magnetic resonance imaging: an emerging tool for evaluating intracranial arterial disease. Stroke 2013;44:287-92 CrossRef Medline

8. Ritz K, Denswil NP, Stam OC, et al. Cause and mechanisms of intracranial atherosclerosis. Circulation 2014;130:1407-14 CrossRef Medline
9. Holmstedt CA, Turan TN, Chimowitz MI. Atherosclerotic intracranial arterial stenosis: risk factors, diagnosis, and treatment. Lancet Neurol 2013;12:1106-14 CrossRef Medline

10. Qureshi AI, Caplan LR. Intracranial atherosclerosis. Lancet 2014; 383:984-98 CrossRef Medline

11. Obusez EC, Hui F, Hajj-Ali RA, et al. High-resolution MRI vessel wall imaging: spatial and temporal patterns of reversible cerebral vasoconstriction syndrome and central nervous system vasculitis. AJNR Am J Neuroradiol 2014;35:1527-32 CrossRef Medline

12. Choi YJ, Jung SC, Lee DH. Vessel wall imaging of the intracranial and cervical carotid arteries. J Stroke 2015;17:238-55 CrossRef Medline

13. Edjlali M, Gentric JC, Régent-Rodriguez C, et al. Does aneurysmal wall enhancement on vessel wall MRI help to distinguish stable from unstable intracranial aneurysms? Stroke 2014;45:3704-06 CrossRef Medline

14. Nagahata S, Nagahata M, Obara M, et al. Wall enhancement of the intracranial aneurysms revealed by magnetic resonance vessel wall imaging using three-dimensional turbo spin-echo sequence with motion-sensitized driven-equilibrium: a sign of ruptured aneurysm? Clin Neuroradiol 2016;26:277-83 CrossRef Medline

15. Lindenholz A, van der Kolk AG, Zwanenburg JJM, et al. The use and pitfalls of intracranial vessel wall imaging: how we do it. Radiology 2018;286:12-28 CrossRef Medline

16. Zhu $\mathrm{C}$, Haraldsson $\mathrm{H}$, Tian $\mathrm{B}$, et al. High resolution imaging of the intracranial vessel wall at 3 and $7 \mathrm{~T}$ using $3 \mathrm{D}$ fast spin echo MRI. MAGMA 2016;29:559-70 CrossRef Medline

17. Wang J, Helle M, Zhou Z, et al. Joint blood and cerebrospinal fluid suppression for intracranial vessel wall MRI. Magn Reson Med 2016; 75:831-38 CrossRef Medline

18. Yang H, Zhang X, Qin Q, et al. Improved cerebrospinal fluid suppression for intracranial vessel wall MRI. J Magn Reson Imaging 2016;44:665-72 CrossRef Medline

19. Qiao Y, Steinman DA, Qin Q, et al. Intracranial arterial wall imaging using three-dimensional high isotropic resolution black blood MRI at 3.0 Tesla. J Magn Reson Imaging 2011;34:22-30 CrossRef Medline

20. Zhang $\mathrm{L}$, Zhang $\mathrm{N}$, Wu J, et al. High resolution three dimensional intracranial arterial wall imaging at $3 \mathrm{~T}$ using $\mathrm{T} 1$ weighted SPACE. Magn Reson Imaging 2015;33:1026-34 CrossRef Medline

21. Xie Y, Yang Q, Xie G, et al. Improved black-blood imaging using DANTE-SPACE for simultaneous carotid and intracranial vessel wall evaluation. Magn Reson Med 2016;75:2286-94 CrossRef Medline

22. Li ML, Xu YY, Hou B, et al. High-resolution intracranial vessel wall imaging using 3D CUBE T1 weighted sequence. Eur J Radiol 2016; 85:803-07 CrossRef Medline

23. Dieleman N, Yang W, van der Kolk AG, et al. Qualitative evaluation of a high-resolution 3D multi-sequence intracranial vessel wall protocol at 3 Tesla MRI. PLoS One 2016;11:e0160781 CrossRef Medline

24. Dieleman N, Yang W, Abrigo JM, et al. Magnetic resonance imaging of plaque morphology, burden, and distribution in patients with symptomatic middle cerebral artery stenosis. Stroke 2016;47:1797802 CrossRef Medline

25. Klein S, Staring M, Murphy K, et al. Elastix: a toolbox for intensitybased medical image registration. IEEE Trans Med Imaging 2010;29: 196-205 CrossRef Medline

26. Swartz RH, Bhuta SS, Farb RI, et al. Intracranial arterial wall imaging using high-resolution 3-Tesla contrast-enhanced MRI. Neurology 2009;72:627-34 CrossRef Medline

27. Fan Z, Yang Q, Deng Z, et al. Whole-brain intracranial vessel wall imaging at 3 Tesla using cerebrospinal fluid-attenuated T1weighted 3D turbo spin echo. Magn Reson Med 2017;77:1142-50 CrossRef Medline

28. Harteveld AA, van der Kolk AG, van der Worp HB, et al. High-resolution intracranial vessel wall MRI in an elderly asymptomatic population: comparison of 3T and 7T. Eur Radiol 2017;27:1585-95 CrossRef Medline 
29. Li L, Miller KL, Jezzard P. DANTE-prepared pulse trains: a novel approach to motion-sensitized and motion-suppressed quantitative magnetic resonance imaging. Magn Reson Med 2012;68:1423-38 CrossRef Medline

30. Edelman RR, Chien D, Kim D. Fast selective black blood MR imaging. Radiology 1991;181:655-60 CrossRef Medline

31. van der Kolk AG, Hendrikse J, Brundel M, et al. Multi-sequence whole-brain intracranial vessel wall imaging at 7.0 Tesla. Eur Radiol 2013;23:2996-3004 CrossRef Medline

32. Mugler JP 3rd. Optimized three-dimensional fast-spin-echo MRI. J Magn Reson Imaging 2014;39:745-67 CrossRef Medline

33. Dieleman N, van der Kolk AG, van Veluw SJ, et al. Patterns of intracranial vessel wall changes in relation to ischemic infarcts. Neurology 2014;83:1316-20 CrossRef Medline

34. Antiga L, Wasserman BA, Steinman DA. On the overestimation of early wall thickening at the carotid bulb by black blood MRI, with implications for coronary and vulnerable plaque imaging. Magn Reson Med 2008;60:1020-28 CrossRef Medline

35. Xu WH, Li ML, Gao S, et al. In vivo high-resolution MR imaging of symptomatic and asymptomatic middle cerebral artery atherosclerotic stenosis. Atherosclerosis 2010;212:507-11 CrossRef Medline

36. Wiesinger F, Van de Moortele PF, Adriany G, et al. Potential and feasibility of parallel MRI at high field. NMR Biomed 2006;19: 368-78 CrossRef Medline

37. Lustig M, Donoho D, Pauly JM. Sparse MRI: The application of compressed sensing for rapid MR imaging. Magn Reson Med 2007;58: 1182-95 CrossRef Medline

38. $\mathrm{Li} \mathrm{B}, \mathrm{Li} \mathrm{H}, \mathrm{Li}$ J, et al. Relaxation enhanced compressed sensing threedimensional black-blood vessel wall MR imaging: preliminary studies. Magn Reson Imaging 2015;33:932-38 CrossRef Medline

39. Bos D, van der Rijk MJ, Geeraedts TE, et al. Intracranial carotid artery atherosclerosis: prevalence and risk factors in the general population. Stroke 2012;43:1878-84 CrossRef Medline

40. Homburg PJ, Plas GJ, Rozie S, et al. Prevalence and calcification of intracranial arterial stenotic lesions as assessed with multidetector computed tomography angiography. Stroke 2011;42:1244-50 CrossRef Medline 